\title{
Topological Derivative for Fast Imaging of Two-Dimensional Thin Dielectric Inclusions in The Wave Propagation Environment
}

\author{
Won-Kwang Park
}

\begin{abstract}
In this paper, we consider the topological derivative concept for developing a fast imaging algorithm of thin inclusions with dielectric contrast with respect to an embedding homogeneous domain with a smooth boundary. The topological derivative is evaluated by applying asymptotic expansion formulas in the presence of small, perfectly conducting cracks. Through the careful derivation, we can design a one-iteration imaging algorithm by solving an adjoint problem. Numerical experiments verify that this algorithm is fast, effective, and stable.
\end{abstract}

Key words: Asymptotic Expansion Formula, Non-Iterative Imaging, Thin Dielectric Inclusions, Topological Derivative.

\section{Introduction}

The inverse scattering problem of imaging the unknown shape of thin penetrable inclusions or of perfectly conducting cracks from measured scattered data is a difficult problem due to ill-posedness and inherent non-linearity. Nevertheless, it is also an interesting one that arises in a number of fields, such as physics, medical science, and material engineering.

Over a number of decades, various imaging algorithms have been developed, many of them based on the Newton-type iteration schemes [1 4]. Nevertheless, the guarantee of a successful imaging performance requires a good initial guess that is close to the unknown target. Without this, issue such as non-convergence, the occurrence of several minima, and large computational costs may arise. These schemes require suitable regularization terms that depend on the problem at hand.

For this reason, various non-iterative algorithms are also developed as alternatives; for example, linear sampling [5, 6], Multiple Signal Classification (MUSIC) [7, $8]$, and multi-frequency methods $[9,10]$. These appear to be fast and robust, and easily extend to the case of several disconnected inclusions in the same domain. Although the results obtained do not guarantee the true shape of inclusions, they could be good initial guesses for Newton-type algorithms. Unfortunately, the successful application of these algorithms requires a large number of incident fields with various directions and corresponding scattered fields are needed. Therefore, to be effective, the non-iterative imaging algorithm that is developed requires a small number of these fields.
In this paper, we develop an alternative non-iterative algorithm that is based on the topological derivative concept for imaging of thin dielectric inclusion from the measured scattered field at a boundary induced from an incident field. For this purpose, the asymptotic expansion formula due to the presence of a small (perfectly conducting) crack is applied to express the topological derivative.

This paper organized as follows. In section 2, the twodimensional direct scattering problem is sketched and the asymptotic formula due to the small crack is introduced. Discussion of the topological derivative concept is considered in section 3 . In section 4 , numerical simulations for demonstrating the performance of the algorithm are illustrated. A short conclusion appears in section 5. In the appendix, a rigorous proof of theorem 1 is explained.

\section{II . Direct Scattering Problems \& Asymptotic Formula}

We consider the two-dimensional electromagnetic scattering from a thin, curve-like inclusion within a homogeneous domain $\Omega$. Assume that this domain contains a thin inclusion, denoted as $\Gamma$, which is localized in the neighborhood of a curve. That is,

$$
\Gamma=\{x+\eta n(x): x \in \sigma, \eta \in(-h, h)\}
$$

where the $\sigma$ is a simple, smooth curve completely embedded in $\Omega, n(x)$ is the unit normal to $\sigma$ at $x$, and $h$ is a strictly positive constant that specifies the thickness of the inclusion (small with respect to the wavelength $\lambda$ ). Throughout this paper, we denote $x, y$ and $z$ are two-

Manuscript received November 26, 2010 ; revised February 15, 2011. (ID No. 20101126-03J)

Department of Mathematics, The College of Natural Sciences, Kookmin University, Seoul, Korea.

Corresponding Author : Won-Kwang Park (e-mail : parkwk@kookmin.ac.kr) 
dimensional vectors.

Assume that all materials are fully characterized by their dielectric permittivity at a given frequency $\omega$. Let $\varepsilon_{0}$ and $\varepsilon$ denote the permittivity of the domain $\Omega$ and thin inclusion $\Gamma$, respectively. Then, one can define the piecewise constant dielectric permittivity as:

$$
\varepsilon(x)=\left\{\begin{array}{lll}
\varepsilon & \text { for } & x \in \Gamma \\
\varepsilon_{0} & \text { for } & x \in \Omega \backslash \bar{\Gamma} .
\end{array}\right.
$$

For the sake of simplicity, throughout this paper, we set $\varepsilon_{0}=1$.

To derive the topological derivative, we will apply asymptotic expansion formulas that take into account the existence of a small crack. For this purpose, we assume that $\Omega$ contains a small, perfectly conducting crack $\Sigma=$ $\left\{z=\left(z_{1}, z_{2}\right):-\rho \leq x \leq \rho\right\}$, where $\rho$ is a small, positive constant that specifies the length of $\Sigma$.

Let $u(x)$ and $v(x)$ be the time-harmonic total field that satisfies the Helmholtz equation in the presence of small crack $\Sigma$ :

$$
\left\{\begin{array}{lll}
\Delta u(x)+\omega^{2} u(x)=0 & \in & \Omega \backslash \bar{\Sigma} \\
u(x)=0 & \text { on } & \Sigma \\
\frac{\partial u}{\partial \nu}(x)=g(x) & \text { on } & \partial \Omega
\end{array}\right.
$$

and

$$
\left\{\begin{array}{lll}
\Delta v(x)+\omega^{2} v(x)=0 & \in & \Omega \backslash \bar{\Sigma} \\
v(x)=0 & \text { on } & \Sigma \\
v(x)=f(x) & \text { on } & \partial \Omega
\end{array}\right.
$$

and let $u_{0}(x)$ and $v_{0}(x)$ be the solution of equations (1) and (2) without $\Sigma$, respectively. Then, the asymptotic expansion formula in the presence of the small crack can be written as [11]:

$$
\begin{aligned}
& u(x)-u_{0}(x)=\frac{2 \pi u_{0}(z)}{\ln (\rho / 2)} N(x, z)+O\left(\rho^{2}\right) \\
& \frac{\partial v}{\partial \nu}(x)-\frac{\partial v_{0}}{\partial \nu}(x)=\frac{2 \pi v_{0}(z)}{\ln (\rho / 2)} \frac{\partial D(x, z)}{\partial \nu(x)}+O\left(\rho^{2}\right)
\end{aligned}
$$

for $x \in \partial \Omega$, where $N(x, z)$ and $D(x, z)$ is Neumann and Dirichlet function for $\Omega$, the solution to:

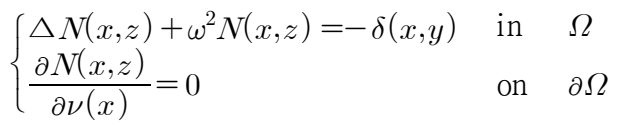

and

$$
\left\{\begin{array}{lll}
\triangle D(x, z)+\omega^{2} D(x, z)=-\delta(x, y) & \text { in } & \Omega \\
D(x, z)=0 & \text { on } & \partial \Omega,
\end{array}\right.
$$

respectively.

\section{Topological Derivative Concept}

The topological derivative measures the influence of creating a small crack (or small ball, inclusions, etc.) at a point inside the domain $\Omega$. Mathematically, the topological derivative $d_{T} J(z)$ of a function $J(\Omega)$ at $z \in \Omega$ can be defined by

$$
J(\Omega \backslash \Sigma)=J(\Omega)+\varphi(\rho) d_{T} J(z)+o(\varphi(\rho)),
$$

where the function $\varphi(\rho) \rightarrow 0$ as $\rho \rightarrow 0[12] \sim[16]$.

Suppose that $\Omega$ contains a small crack $\Sigma, g(x)$ denotes the boundary condition on $\partial \Omega$ and $u_{\Gamma}(x)$ is the solution to the problem in the presence of a thin inclusion:

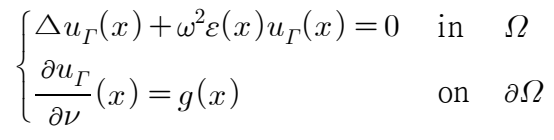

With this, construct $u_{N}(x)$ and $u_{D}(x)$ as the solutions to the following problems in the absence of inclusion:

$$
\begin{cases}\Delta u_{N}(x)+\omega^{2} u_{N}(x)=0 & \text { in } \quad \Omega \\ \frac{\partial u_{N}}{\partial \nu}(x)=g(x) & \text { on } \quad \partial \Omega\end{cases}
$$

and

$$
\left\{\begin{array}{lll}
\Delta u_{D}(x)+\omega^{2} u_{D}(x)=0 & \text { in } & \Omega \\
u_{D}(x)=u_{\Gamma}(x) & \text { on } & \partial \Omega
\end{array}\right.
$$

respectively. Let us define following discrepancy function:

$$
J(\Omega)=\frac{1}{2} \int_{\partial \Omega}\left(\left|\frac{\partial u_{D}}{\partial \nu}(x)-g(x)\right|^{2}+\left|u_{N}(x)-u_{\Gamma}(x)\right|^{2}\right) d S .
$$

Then, we can obtain the following result:

Theorem 1. The topological derivative $d_{T} J(z)$ of the discrepancy function $J(\Omega)$ satisfies

$$
J(\Omega \backslash \Sigma)=J(\Omega)+\varphi(\rho) d_{T} J(z)+o(\varphi(\rho)),
$$

where

$$
\varphi(\rho)=\frac{2 \pi}{\ln (\rho / 2)}
$$

and

$$
d_{T} J(z)=\operatorname{Re}\left[v_{N}(z) u_{N}(z)-v_{D}(z) u_{D}(z)\right] .
$$

Here, adjoint states $v_{N}(z)$ and $v_{D}(z)$ are defined as the solution to

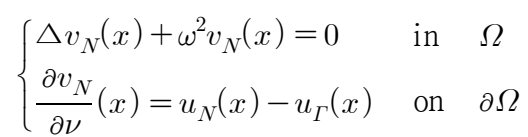

and

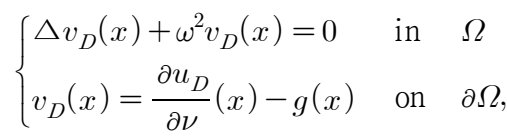


respectively.

\section{Proof. See Appendix.}

The points where the topological derivative is the most negative value are expected to be approximately on the supporting curve $\sigma$ so that this quickly yields an outline of shape of the unknown thin dielectric inclusion $\Gamma$.

Remark 1. Instead of creating a small, perfectly conducting crack in the domain $\Omega$, one can also create a small ball of radius $\rho$. Derivation of the topological derivative and corresponding numerical result can be found in the recent work [16].

\section{Numerical Simulations}

We now present some results of numerical simulations using the topological derivative $d_{T} J(z)$ to image unknown thin dielectric inclusions in $\Omega$. For this purpose, we choose the homogeneous domain $\Omega$ as a two-dimensional unit circle centered at the origin and two thin inclusions $\Gamma_{j}$ for illustration:

$$
\Gamma_{j}=\left\{x+\eta n(x): x \in \sigma_{j}, \eta \in(-0.02,0.02)\right\}
$$

with supporting curves $\sigma_{j}$ represented as:

$$
\begin{aligned}
& \sigma_{1}=\left\{\left(s-0.2,-0.5 s^{2}+0.5\right): s \in[-0.5,0.5]\right\} \\
& \sigma_{2}=\left\{\left(s+0.2, s^{3}+s^{2}-0.6\right): s \in[-0.5,0.5]\right\}
\end{aligned}
$$

and we denote $\varepsilon_{j}$ be the permittivity of $\Gamma_{j}$. Since we choose $\varepsilon_{0}=1$, the applied frequency reads as $\omega=2 \pi / \lambda$, at wavelength $\lambda$, i.e., if we consider the plane wave illumination, the boundary conditions in equations (1), (3) and (4) can be read as (see Fig. 1):

$g(x)=\frac{\partial u}{\partial \nu}(x)=i \omega \theta \cdot \nu(x) \exp (i \omega \theta \cdot x), x \in \partial \Omega$.

Notice that throughout this paper, the wavelength is chosen as 0.5 , i.e., $\lambda=0.5$.

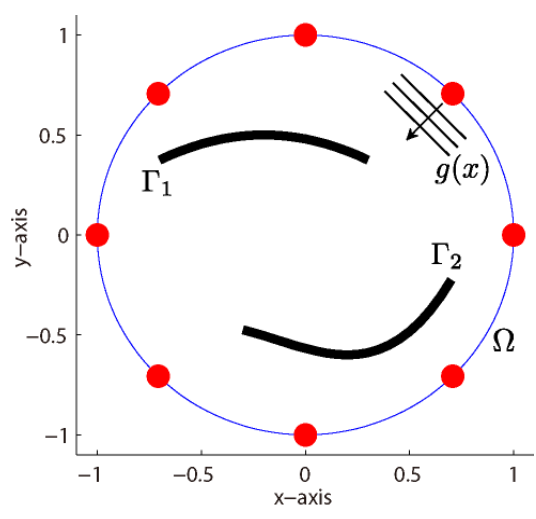

Fig. 1. Test configuration.

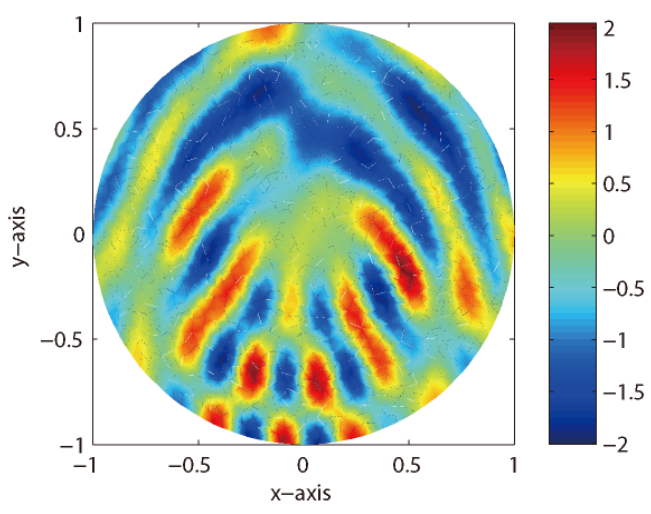

Fig. 2. $d_{T} J(z)$ for $\Gamma_{1}$ with 1 direction.

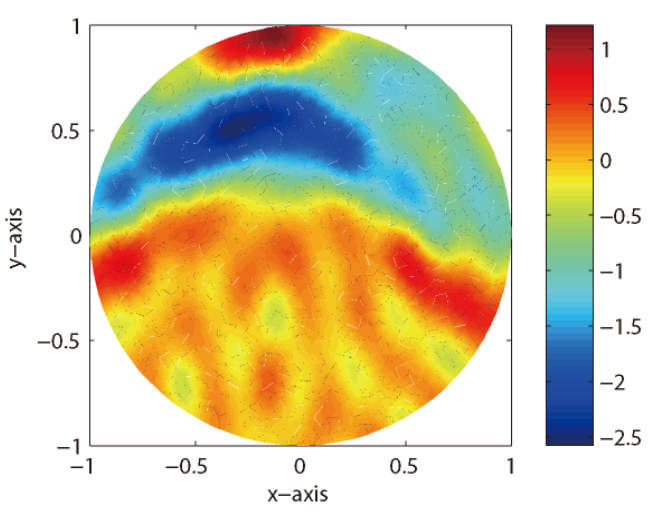

Fig. 3. $d_{T} J(z)$ for $\Gamma_{1}$ with 2 directions.

Remark 2. For a successful application of the imaging algorithm, one must apply adequate numbers of different incident directions $\theta$. For example, Fig. 2 shows the map of $d_{T} J(z)$ with one incident direction $\theta=(1,0)$. In this result, it is very difficult to recognize the shape of $\Gamma_{1}$. However, when we apply two different incident directions $\theta=(1,0)$ and $\theta=(-1,0)$, we obtain a more acceptable result, as seen in Fig. 3. Based on these experiments, we apply 8 different number of different incident directions to obtain a good result, as follows:

$$
\theta=\left(\cos \frac{n \pi}{4}, \sin \frac{n \pi}{4}\right), n=1,2, \cdots, 8 .
$$

For the first example, let us consider the imaging of $\Gamma_{1}$ with $\varepsilon_{1}=5$ Fig. 4 is the map of $d_{T} J(z)$ for all $z \in \Omega$. In this example, one can easily notice that the point where the topological derivative is the most negative value appears in the neighborhood of $\Gamma_{1}$.

Next, Fig. 5 shows the map of $d_{T} J(z)$ when the thin inclusion is $\Gamma_{2}$ with $\varepsilon_{2}=5$ Although, part of $\Gamma_{2}$ is not apparent, the result obtained is close to the $\Gamma_{2}$.

Mathematical configurations, derivation of topological derivative, and numerical analysis could be extended to the case of multiple thin inclusions, say, $\Gamma_{M}=\Gamma_{1} \cup \Gamma_{2}$. In 
Fig. 6, a good imaging result appears when permittivities of $\Gamma_{1}$ and $\Gamma_{2}$ are $\varepsilon_{1}=\varepsilon_{2}=5$. However, in Fig. 7, one can recognize that when one inclusion (say $\Gamma_{2}, \varepsilon_{2}=5$ ) has a much smaller value of permittivity than another (say $\Gamma_{1}, \varepsilon_{1}=10$ ), the first inclusion appeared with much smaller magnitude than the second.

Finally, in order to show the robustness of the proposed algorithm, we added a white Gaussian random noise with a $20 \mathrm{~dB}$ signal-to-noise ratio (SNR) to the measured boundary data $u_{\Gamma}(x)$ in equation (3). Fig. 8 and Fig. 9 illustrate the results when we add Gaussian random noise for $\Gamma_{2}$ and $\Gamma_{M}$, respectively with $\varepsilon_{1}=10$ and $\varepsilon_{2}=5$. By comparing with Fig. 5 and Fig. 7, we observe that proposed method is robust even in the presence of random noise.

\section{Concluding Remarks}

A non-iterative algorithm based on the topological derivative concept has been investigated for imaging thin dielectric curve-like inclusions embedded in a homogeneous domain $\Omega$. Results show that this approach is fast, effective, and stable.

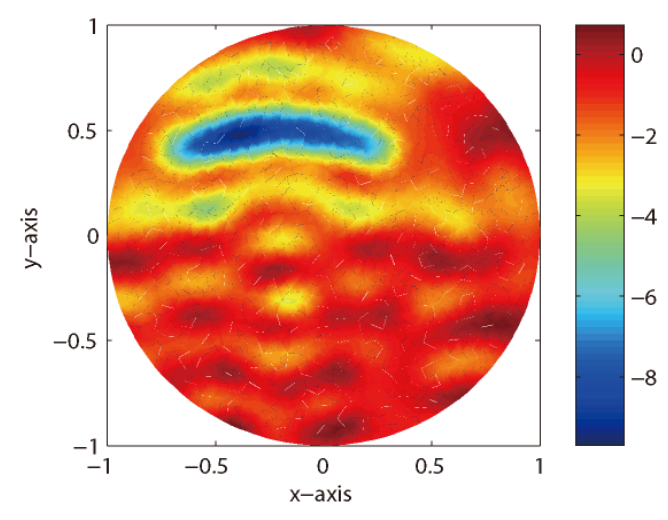

Fig. 4. $d_{T} J(z)$ for $\Gamma_{1}$ with 8 directions.

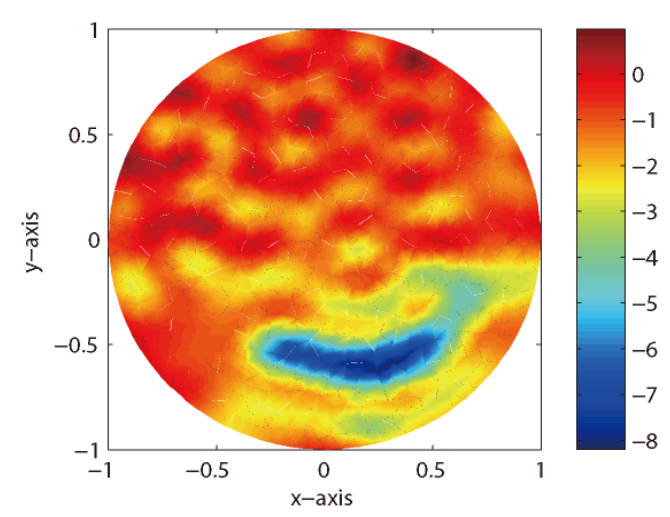

Fig. 5. $d_{T} J(z)$ for $\Gamma_{2}$ with 8 directions.

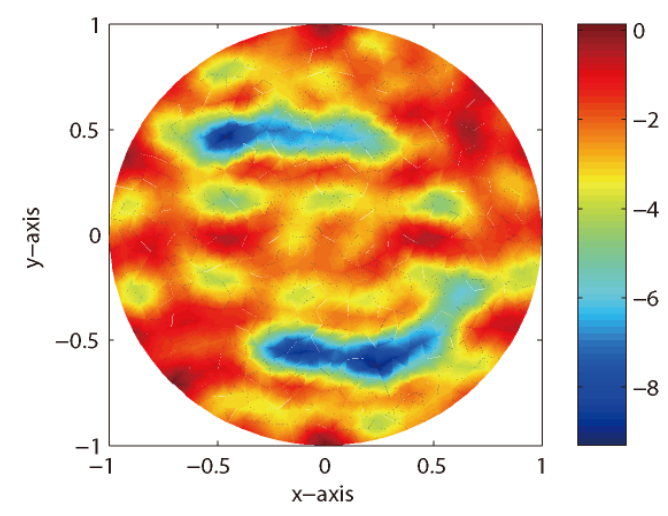

Fig. 6. $d_{T} J(z)$ for $\Gamma_{M}$ with same permittivities.

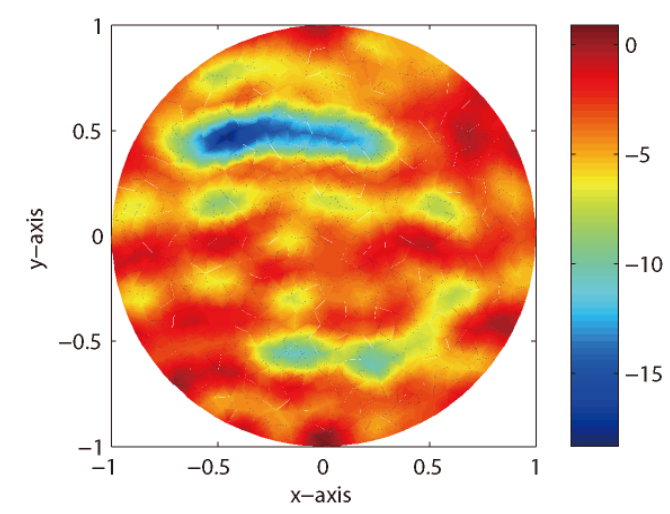

Fig. 7. $d_{T} J(z)$ for $\Gamma_{M}$ with different permittivities.

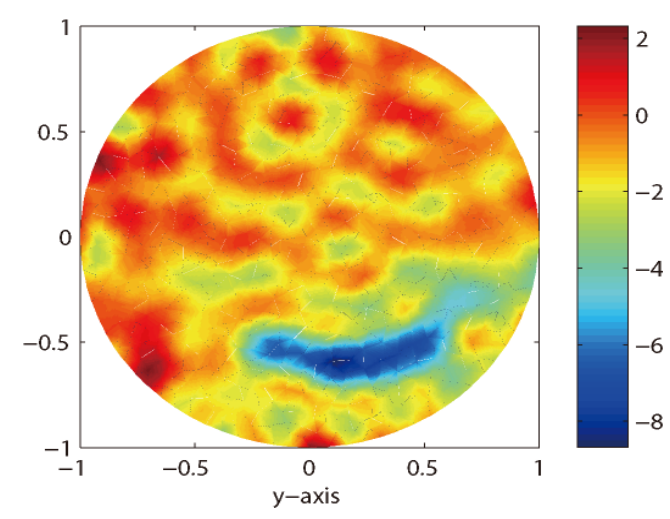

Fig. 8. $d_{T} J(z)$ for $\Gamma_{2}$ with random noise.

In this paper and in recent work [16], we have only considered the dielectric contrast case. However, the analysis could be extended to a purely magnetic contrast between inclusions and embedding domain case.

Recently, we derived the topological derivative for a purely magnetic contrast case. If we define the piecewise constant magnetic permeability as:

$$
\mu(x)=\left\{\begin{array}{lll}
\mu & \text { for } & x \in \Gamma \\
\mu_{0} & \text { for } & x \in \Omega \backslash \bar{\Gamma}
\end{array}\right.
$$




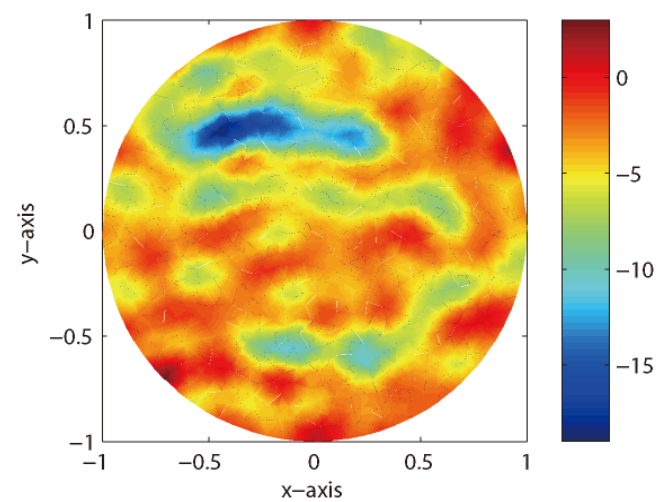

Fig. 9. $d_{T} J(z)$ for $\Gamma_{M}$ with random noise.

then the topological derivative $d_{T} J(z)$ is of the following form:

$$
\operatorname{Re}\left[\nabla v_{D}(z) \cdot \nabla u_{D}(z)-\nabla v_{N}(z) \cdot \nabla u_{N}(z)\right]
$$

when we create a small ball of radius $\rho$. A rigorous derivation of topological derivative and corresponding numerical simulations will be presented in forthcoming work.

\section{Appendix: Proof of Theorem 1}

In order to derive the topological derivative, let us create a small, perfectly conducting crack $\Sigma$ at a certain point $z$ inside the domain $\Omega$ and denote $u_{N}^{\Sigma}(x)$ and $u_{D}^{\Sigma}(x)$ satisfies equations (1) and (2), respectively.

By applying asymptotic expansion formulas, we can examine the relationship between $J(\Omega \backslash \Sigma)$ and $J(\Omega)$ as follows:

$$
\begin{aligned}
& J(\Omega \backslash \Gamma) \\
= & \frac{1}{2} \int_{\partial \Omega}\left(\left|\frac{\partial u_{D}^{\Sigma}}{\partial \nu}(x)-g(x)\right|^{2}+\left|u_{N}^{\Sigma}(x)-u_{\Gamma}(x)\right|^{2}\right) d S \\
= & \frac{1}{2} \int_{\partial \Omega}\left(\left|\frac{\partial u_{D}}{\partial \nu}(x)-g(x)\right|^{2}+\left|u_{N}(x)-u_{\Gamma}(x)\right|^{2}\right) d S \\
& +\int_{\partial \Omega}\left(\frac{\partial u_{D}}{\partial \nu}(x)-g(x)\right)\left(\frac{\partial u_{D}^{\Sigma}}{\partial \nu}(x)-\frac{\partial u_{D}}{\partial \nu}(x)\right) d S \\
& +\int_{\partial \Omega}\left(u_{N}(x)-u_{\Gamma}(x)\right)\left(\frac{u_{N}^{\Sigma}(x)-u_{N}(x)}{u^{2}}\right) d S+o\left(\rho^{2}\right) \\
= & J(\Omega)+J_{1}(z)+J_{2}(z)+O\left(\rho^{2}\right),
\end{aligned}
$$

where

$$
J_{1}(z)=\int_{\partial \Omega}\left(u_{N}(x)-u_{\Gamma}(x)\right)\left(\overline{u_{N}^{\Sigma}(x)-u_{N}(x)}\right) d S
$$

and

$$
J_{2}(z)=\int_{\partial \Omega}\left(\frac{\partial u_{D}}{\partial \nu}(x)-g(x)\right)\left(\overline{\frac{\partial u_{D}^{\Sigma}}{\partial \nu}(x)-\frac{\partial u_{D}}{\partial \nu}(x)}\right) d S .
$$

Therefore, we can obtain the topological derivative $d_{T} J(z)$ through the evaluation of $J_{1}(z)$ and $J_{2}(z)$.
First, from the boundary condition of equation (6) and asymptotic expansion formula, we can compute

$$
\begin{aligned}
J_{1}(z) & =\int_{\partial \Omega}\left(u_{N}(x)-u_{\Gamma}(x)\right)\left(\overline{u_{N}^{\Sigma}(x)-u_{N}(x)}\right) d S \\
& =\int_{\partial \Omega} \frac{\partial v_{N}}{\partial \nu}(x)\left(\overline{u_{N}^{\Sigma}(x)-u_{N}(x)}\right) d S \\
& =\frac{2 \pi v_{0}(z)}{\ln (\rho / 2)} \int_{\partial \Omega} \frac{\partial v_{N}}{\partial \nu}(x)\left(\overline{u_{N}(z) N(x, z)}\right) d S .
\end{aligned}
$$

Then, applying integration by parts yields:

$$
\begin{aligned}
& \int_{\partial \Omega} \frac{\partial v_{N}}{\partial \nu}(x)\left(\overline{u_{N}(z) N(x, z)}\right) d S \\
= & \int_{\Omega} \Delta v_{N}(x) \overline{u_{N}(z) N(x, z)} d x \\
& +\int_{\Omega} \nabla v_{N}(x) \cdot \overline{u_{N}(z) \nabla N(x, z)} d x \\
= & \int_{\Omega}\left[\Delta v_{N}(x)+\omega^{2} v_{N}(x)\right] \overline{u_{N}(z) N(x, z)} d x \\
& -\int_{\Omega} v_{N}(x) \overline{u_{N}(z) \Delta N(x, z)} d x \\
& -\int_{\Omega}^{\omega^{2} v_{N}(x) \overline{u_{N}(z) N(x, z)}} d x \\
= & -\int_{\Omega}^{v_{N}(x) \overline{u_{N}(z)\left[N(x, z)+\omega^{2} N(x, z)\right]}} d x \\
= & \int_{\Omega} v_{N}(x) \overline{u_{N}(z) \delta(x, z)} d x \\
= & v_{N}(z) \overline{u_{N}(z)} .
\end{aligned}
$$

Therefore, we can obtain:

$J_{1}(z)=\frac{2 \pi v_{0}(z)}{\ln (\rho / 2)} v_{N}(z) \overline{u_{N}(z)}$.

Next, from the boundary condition of equation (7) and asymptotic expansion formula, we can compute

$$
\begin{aligned}
J_{2}(z) & =\int_{\partial \Omega}\left(\frac{\partial u_{D}}{\partial \nu}(x)-g(x)\right)\left(\overline{\frac{\partial u_{D}^{\Sigma}}{\partial \nu}(x)-\frac{\partial u_{D}}{\partial \nu}(x)}\right) d S \\
& =\int_{\partial \Omega} v_{D}(x)\left(\overline{\frac{\partial u_{D}^{\Sigma}}{\partial \nu}(x)-\frac{\partial u_{D}}{\partial \nu}(x)}\right) d S \\
& =\frac{2 \pi v_{0}(z)}{\ln (\rho / 2)} \int_{\partial \Omega} v_{D}(x) \overline{u_{D}(z) \frac{\partial D(x, z)}{\partial \nu(x)}} d S .
\end{aligned}
$$

Then, applying integration by parts yields:

$$
\begin{aligned}
& \int_{\partial \Omega} v_{D}(x) \overline{u_{D}(z) \frac{\partial D(x, z)}{\partial \nu(x)}} d S \\
= & \int_{\Omega} v_{D}(x) \overline{u_{D}(z) \Delta D(x, z)} d x \\
& +\int_{\Omega}^{\Omega} \nabla v_{D}(x) \cdot \overline{u_{D}(z) \nabla D(x, z)} d x \\
= & \int_{\Omega} v_{D}(x)\left[\overline{u_{D}(z) \Delta D(x, z)+u_{D}(z) \omega^{2} D(x, z)}\right] d x \\
& -\int_{\Omega}^{\Omega} \Delta v_{D}(x) \overline{u_{D}(z) D(x, z)} d x \\
& -\int_{\Omega}^{\Omega} \Delta v_{D}(x) \overline{u_{D}(z) \omega^{2} D(x, z)} d x \\
= & -\int_{\Omega}^{v_{D}(x) \overline{u_{D}(z) \delta(x, z)}} d x \\
& -\int_{\Omega}\left[\Delta v_{D}(x)+\omega^{2} v_{D}(x)\right] \overline{u_{D}(z) D(x, z)} d x \\
= & -v_{D}(z) \overline{u_{D}(z)} .
\end{aligned}
$$


Therefore,

$$
J_{2}(z)=-\frac{2 \pi v_{0}(z)}{\ln (\rho / 2)} v_{D}(z) \overline{u_{D}(z)} .
$$

Finally, by taking real parts of sum of equations (8) and (9), we can obtain the desired result. This ends the proof.

\section{References}

[1] D. Alvarez, O. Dorn, N. Irishina, and M. Moscoso, "Crack reconstruction using a level-set strategy," $J$. Comput. Phys., vol. 228, pp. 5710-5721, 2009.

[2] F. Delbary, K. Erhard, R. Kress, R. Potthast and J. Schulz, "Inverse electromagnetic scattering in a twolayered medium with an application to mine detection," Inverse Problems, vol. 24, 015002, 2008.

[3] O. Dorn, D. Lesselier, "Level set methods for inverse scattering," Inverse Problems, vol. 22, R67-R131, 2006.

[4] W.-K. Park, D. Lesselier, "Reconstruction of thin electromagnetic inclusions by a level set method," Inverse Problems, vol. 25, 085010, 2009.

[5] M. Cheney, "The linear sampling method and the MUSIC algorithm," Inverse Problems, vol. 17, pp. 591-595, 2001.

[6] D. Colton, H. Haddar, and P. Monk, "The linear sampling method for solving the electromagnetic inverse scattering problem," SIAM J. Sci. Comput., vol. 24, pp. 719-731, 2002.

[7] W.-K. Park, D. Lesselier, "Electromagnetic MUSICtype imaging of perfectly conducting, arc-like cracks at single frequency," J. Comput. Phys., vol. 228, pp. 8093-8111, 2009.

\section{Won-Kwang Park}

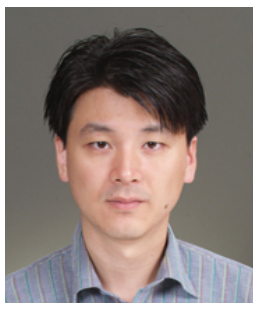

received his B.S. degree in Mathematical Education from Kookmin University, Seoul, Korea in 2000, M.S. degree in Mathematics from Yonsei University, Seoul, Korea in 2004, and Ph.D. degree in Applied Mathematics (Centre de Mathématiques Appliquées) from École Polytechnique, Palaiseau, France in 2009. After graduation, he joined the Laboratoire des Signaux et Systmès (LSS), École Supérieure d'Électricité (Supélec), Gif-sur-Yvette, France and the institute for mathematics and scientific computing, University of Graz, Austria, as a post doc. researcher in 2009. Currently, he is a full-time lecturer of Department of Mathematics in Kookmin University. His main research area includes partial differential equations, inverse problems, electromagnetics, and scientific computing.
[8] W.-K. Park, D. Lesselier, "MUSIC-type imaging of a thin penetrable inclusion from its far-field multi-static response matrix," Inverse Problems, vol. 25, 075002, 2009.

[9] W.-K. Park, "Non-iterative imaging of thin electromagnetic inclusions from multi-frequency response matrix," Progress in Electromagnetic Research, vol. 106, pp. 225-241, 2010.

[10] W.-K. Park, "On the imaging of thin dielectric inclusions buried within a half-space," Inverse Problems, vol. 26, 074008, 2010.

[11] H. Ammari, H. Kang, H. Lee, and W.-K. Park, "Asymptotic imaging of perfectly conducting cracks," SIAM J. Sci. Comput., vol. 32, pp. 894-922, 2010.

[12] D. Auroux, M. Masmoudi, "Image processing by topological asymptotic analysis," ESAIM Proc., vol. 26, pp. 24-44, 2009.

[13] A. Capiro, M.-L. Rapun, "Solving inhomogeneous inverse problems by topological derivative methods," Inverse Problems, vol. 24, 045014, 2008.

[14] H. A. Eschenauer, V. V. Kobelev, and A. Schumacher, "Bubble method for topology and shape optimization of structuresn," Struct. Optim., vol. 8, pp. 42-51, 1994.

[15] J. Sokolowski, A. Zochowski, "On the topological derivative in shape optimization," SIAM J. Control Optim., vol. 37, pp. 1251-1272, 1999.

[16] W.-K. Park, "On the imaging of thin dielectric inclusions via topological derivative concept," Progress in Electromagnetic Research, vol. 110, pp. 237-252, 2010. 